OPEN ACCESS

Edited by:

Fushun Wang,

Nanjing University of Chinese

Medicine, China

Reviewed by:

Liu Dexiang,

Shandong University, China

Huiling Wang,

Renmin Hospital of Wuhan

University, China

Tijen Utkan,

Kocaeli University, Turkey

*Correspondence:

Weirong $\mathrm{Li}$

liwr@gzucm.edu.cn

Yafei Shi

shiyafei@gzucm.edu.cn

tThese authors have contributed equally to this work and share first authorship

Specialty section:

This article was submitted to Psychopathology,

a section of the journal

Frontiers in Psychiatry

Received: 03 March 2020 Accepted: 05 February 2021

Published: 05 March 2021

Citation:

Huang J, Shen C, Ye R, Shi Y and

Li W (2021) The Effect of Early Maternal Separation Combined With

Adolescent Chronic Unpredictable Mild Stress on Behavior and Synaptic Plasticity in Adult Female Rats.

Front. Psychiatry 12:539299.

doi: 10.3389/fpsyt.2021.539299

\section{The Effect of Early Maternal Separation Combined With Adolescent Chronic Unpredictable Mild Stress on Behavior and Synaptic Plasticity in Adult Female Rats}

\author{
Jiawen Huang ${ }^{1,2 \dagger}$, Chongkun Shen ${ }^{3 \dagger}$, Ran Ye ${ }^{4,5 t}$, Yafei Shi ${ }^{3 *}$ and Weirong $\mathrm{Li}^{1,2 *}$ \\ ${ }^{1}$ Science and Technology Innovation Center, Guangzhou University of Chinese Medicine, Guangzhou, China, ${ }^{2}$ Institute of \\ Clinical Pharmacology, Guangzhou University of Chinese Medicine, Guangzhou, China, ${ }^{3}$ School of Fundamental Medical \\ Science, Guangzhou University of Chinese Medicine, Guangzhou, China, ${ }^{4}$ Heyuan People's Hospital, Heyuan, China, \\ ${ }^{5}$ School of Pharmaceutical Sciences, Guangzhou University of Chinese Medicine, Guangzhou, China
}

Our aims were to evaluate the depression model of early maternal separation (MS) combined with adolescent chronic unpredictable mild stress (CUMS) in female adult SD rats to observe the behavior and the expressions of synaptic proteins in rats and to provide a reference for the screening of antidepressant drug activity. In our study, MS and CUMS were conducted to establish a dual stress model on female rats. Behavioral tests, including the sucrose preference test, open field test, and zero maze test, were used to detect depression-like and anxiety-like behavior of animals. Nissl staining was used to detect the number of neuronal cells in the hippocampus CA1 and DG regions of rats from each group. Synaptophysin (SYN), postsynaptic density-95 (PSD-95), and growth-associated protein-43 (GAP-43) expressions in the hippocampus were detected by western blot. Expression of the hippocampus SYN protein was further detected by immunohistochemistry. Rats in the MS+CUMS group presented more serious depression-like and anxiety-like behavior than in the MS group. Also, few Nissl bodies in the hippocampus CA1 and DG regions, less percentage of SYN-positive cells, and downregulated expressions of SYN, PSD-95, and GAP43 were found in the hippocampus of rats in MS+CUMS group. In conclusion, adult female rats that underwent MS and CUMS performed more critical depression-like and anxiety-like behaviors, and this process may be resulted from synaptic plasticity impairment.

Keywords: maternal separation, depression, anxiety, synaptic plasticity, early stress

\section{INTRODUCTION}

Depression is a common psychiatric disorder with a high incidence, and its pathogenesis is still not clear. The World Health Organization points out that depression would be one of the three major diseases in 2030 (1). However, due to the complexity of the etiology of depression, how to establish an effective model of depression has become one of the key issues when researching depression. Additionally, although the etiology of depression has not been confirmed, most scholars believe that the occurrence of depression is influenced by environment and heredity (2). Berton et al. found 
that genetic factors account for $40-50 \%$ of all factors leading to depression, and the remaining $50-60 \%$ are closely related to life stress in early childhood (3). Another study has demonstrated that the one who has adverse experience at a young age may have a risk of depression 4-fold higher than normal persons (4).

Contemporary studies on depression are mostly in male animals, while female animals are often excluded because of interference of estrogen (5-7). In fact, there are reports that women are twice as likely as men to experience depression and anxiety disorders $(8,9)$. So, it is more valuable to study depression in female rats. In addition, adolescence is the most critical period of physiological and psychological changes, and it plays an indispensable role in neurodevelopment and mental diseases. Maternal separation (MS) is a classical model of early stress study. Moreover, it is known that chronic unpredictable mild stress (CUMS) overcomes the stress habit and is commonly used to simulate depressive behavior (10). We chose female rats with MS combined with CUMS as experimental subjects of early stress. On the one hand, the complexity of the etiology of depression was in our consideration. On the other hand, it could also provide a novel model design for the construction of a new model to explore the impact of early stress on adult depression. As anxiety and depression often occur together (11), we would explore both depression-like and anxiety-like behaviors in our study.

Clinical studies have found that the hippocampus of depressed patients is reduced in volume $(12,13)$. Animal studies have found that neurons and glial atrophy are lost in the hippocampus of depression-like model animals (14). The reason may be that stress not only reduced dendritic spine density and number of branches but also thinned postsynaptic density (15). The hippocampus, a brain structure regulating stress and related to depression (16), is more vulnerable to stimulation damage (17), which may lead to neuron reduction $(18,19)$. Previous studies have shown that depression is closely related to the hippocampus synaptic plasticity $(20,21)$. Menard et al. have shown that the number of synapses in depression animals is significantly reduced, and the residual synaptic structure and function have different degrees of damage (22). Increasing evidence had found that dysregulation of synaptic plasticity is related to depression (21). However, there are so far only a few papers on synaptic plasticity proteins $(2,23)$. Thus, changes in synaptic plasticity proteins in the depression model are the focus of this study. This study provides a basis for research on synaptic plasticity protein-related depression and suggests that drugs may be able to improve depression by changing synaptic plasticity.

Based on the researches above, the stress model of MS, CUMS, and MS combined with CUMS were used in this study. We tried to observe the effects of different stress on the depression-like and anxiety-like behaviors of adult female rats and synaptic plasticity of the hippocampus.

\section{MATERIALS AND METHODS}

\section{Animals and Groups}

A total of 10 Sprague Dawley (SD) pregnant rats were purchased from the Experimental Animal Center of Guangzhou University of Chinese Medicine (China). Animals were housed in a constant animal facility at a temperature of $20-25^{\circ} \mathrm{C}$, relative humidity of $50-60 \%$, food and water ad-libitum, and a $12 \mathrm{~h}$ light/dark cycle. On the day the pups were delivered (PND0), only female pups were selected and then were divided into four groups ( $n=8$ animals/group) randomly: the non-maternal separation (CON group), maternal separation only group (MS group), chronic unpredictable mild stress only group (CUMS group), and maternal separation group plus chronic unpredictable mild stress (MS + CUMS group). The body weight was detected once a week. The study design flowchart is illustrated in Figure 1. After behavioral tests, on PND62, animals were anesthesia with $10 \%$ chloral hydrate, and the brain tissues were removed. All experimental protocols were approved by the Animal Experimental Committee of Guangzhou University of Chinese Medicine (Ethical approval number: 20190605015).

\section{Maternal Separation (MS)}

The protocol of MS we used was established in our previous study (24). In brief, from PND1 to PND21, the pups in the MS group and MS+CUMS group were separated from their mother for a total of 360 min each day (8:00-11:00 a.m. and 14:00-17:00 p.m.). During separation time, these pups were placed into cages filled with cotton for the purpose of maintaining the temperature. Rats in the CON group and CUMS group remained with their mother and did not undergo any interference during the MS period. Since PND21, rats were put into cages without their mother with four pups per cage.

\section{Chronic Unpredictable Mild Stress (CUMS)}

Similar to the previous studies $(25,26)$, from PND28, pups received one of nine trials (Table 1) every day until the end of the whole study (PND61). Each trial was randomly taken every day, and the same trial was not used twice in a row.

\section{Sucrose Preference Test (SPT)}

On PND56, rats were given two bottles of 1\% sucrose solution for $24 \mathrm{~h}$; one bottle was replaced with pure water on PND57, and the position of two bottles was exchanged after $12 \mathrm{~h}$; on PND58, food and water were deprived from rats for $24 \mathrm{~h}$; on PND59, the rats were given a bottle of pure water and a bottle of $1 \%$ sucrose solution. After $2 \mathrm{~h}$, the lost weight of each bottle was recorded to determine the intake of the rats. Sucrose Preference rate $(\%)=$ Sucrose consumption/(Sucrose consumption + water consumption) $* 100 \%$.

\section{Open Field Test (OFT)}

OFT was conducted on PND56. The rats were placed in the center of a $100 \times 100 \times 60 \mathrm{~cm}$ black box, and the movement of each rat in the box was automatically recorded by the videotracking analysis system for $3 \mathrm{~min}$. The time in the central area and the total distance would be used as indicators.

\section{Zero Maze Test (ZMT)}

ZMT was conducted on PND60. The maze (outer diameter: $100 \times 100 \mathrm{~cm}$; inner diameter: $80 \times 80 \mathrm{~cm}$ ) was divided into four quadrants (two are opposing open quadrants and two are opposing closed quadrants). Rats were placed in an open quadrant, and the tracking system was triggered (Shanghai 
PND28-61

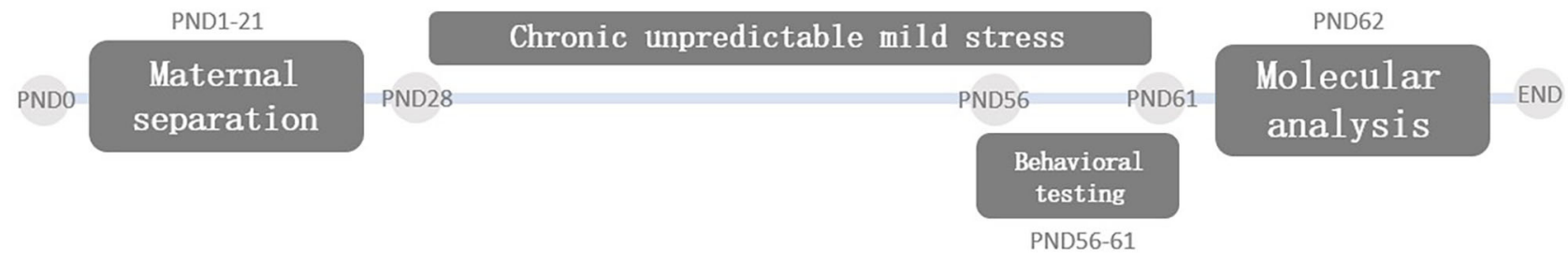

FIGURE 1 | Study design flowchart. PND, postnatal day.

TABLE 1 | Method for CUMS comprises nine trials.

\begin{tabular}{ll}
\hline Trials & \\
\hline 1 & Food deprivation for $24 \mathrm{~h}$ \\
2 & Water deprivation for $24 \mathrm{~h}$ \\
3 & Thermal water stimulation $\left(45^{\circ} \mathrm{C}\right)$ for 5 min \\
4 & Day and night reversal for $24 \mathrm{~h}$ \\
5 & Ice water stimulation $\left(4^{\circ} \mathrm{C}\right)$ for 5 min \\
6 & Cage tilting $45^{\circ}$ for $24 \mathrm{~h}$ \\
7 & Wet padding for $24 \mathrm{~h}$ \\
8 & Crowded squirrel cage for $24 \mathrm{~h}$ \\
9 & Empty water bottle for $2 \mathrm{~h}$ \\
\hline
\end{tabular}

Xinsoft Information Technology Co., Ltd., China). Each rat was tested for $5 \mathrm{~min}$, and the time rat spent in open quadrants would be used as an index.

\section{NissI Staining}

On PND62, brain tissues were removed and then embedded and cut at $5 \mu \mathrm{m}$ thickness. The staining was carried out with Nissl staining solution (Shanghai Biyuntian Biotechnology Co., Ltd., China) for $40 \mathrm{~min}$ at $37^{\circ} \mathrm{C}$ after the sections dewaxing and rehydration. The number of Nissl bodies in the CA1 and DG region was observed under a field of view of a microscope (Leica Microsystems Wetzlar $\mathrm{GmbH}$ ) at $400 \times$ magnification. Select cells that clearly observe the nucleus and nucleolus for statistics.

\section{Immunohistochemistry}

The embedded brain tissues were cut into $5 \mu \mathrm{m}$ sections. Firstly, after dewaxing, the sections were immersed in $0.01 \mathrm{M}$ sodium citrate buffer solution at $95-100^{\circ} \mathrm{C}$ for $15 \mathrm{~min}$ and then incubated with $3 \%$ hydrogen peroxide treatment for $10 \mathrm{~min}$. Secondly, after being incubated with goat serum working solution for $15 \mathrm{~min}$, the sections were incubated with anti-rabbit SYN antibody (Affinity, USA) at $4^{\circ} \mathrm{C}$ overnight. Thirdly, on the following day, the sections were incubated with biotinylated IgG, streptavidin, and $\mathrm{DAB}$ following the DAB detection kit (ZSGB-BIO, China). The sections were digitized and analyzed by a Leica microscope (Leica Microsystems Wetzlar GmbH). Each section was imaged at $400 \times$ magnification and measured the percentage of SYN-positive area in each field of view by quantitative image analysis with ImageJ 1.45 software.

\section{Western Blot}

Total protein of the hippocampus was lysed by using RIPA lysis buffer, and protein concentrations were quantified by using a BCA kit (Beijing Dingguo Changsheng Biotechnology co., Ltd., China). Proteins were separated by sodium dodecyl sulfate polyacrylamide gel electrophoresis (SDS-PAGE), then transferred to PVDF membranes and blocked with 5\% skim milk powder. Antibodies against SYN (Affinity (USA), AF025750; 1:1000), PSD-95 (Affinity (USA), AF5283-50; 1:1000), GAP43 (Affinity (USA), DF7766-50; 1:1000), and Tubulin (Affinity (USA), AF7018-50; 1: 5000) were incubated with membranes overnight at $4{ }^{\circ} \mathrm{C}$. Finally, the IgG-HRP antibody (CST (USA), BST11L22C51, 1:5000) was incubated with membranes for $1 \mathrm{~h}$ at room temperature. After the above steps were completed, membranes were combined with ECL Plus reagent (Beijing Lanjieke Technology Co., Ltd.) for a color reaction. Besides, the gray value was quantified by using Image Lab software.

\section{Statistical Analysis}

SPSS22 software was used to perform analysis and all data are expressed as mean \pm standard error (SEM). One-way analysis of variance (ANOVA) was used for statistical analysis. When the variances were homogeneous, we used an LSD post-hoc test; otherwise, we used Dunnett's T3 test. The results were performed using GraphPad Prism 6.0. $p<0.05$ was considered statistically significant.

\section{RESULTS}

\section{The Effect of MS and/or CUMS on Body Weight}

We observed the effect of MS and CUMS on body weight in this study, including PND28 and PND60 time points (Figure 2). As shown in Figure 2A, after MS for 28 days, rats in the MS group showed no significant influence on body weight compared with the non-MS group. After CUMS, rats in CUMS group $\left[F_{(3,31)}=34.76, p<0.01\right]$ and MS+CUMS group $\left[F_{(3,31)}=34.76, p<0.01\right]$ showed lower body weight than rats in the control group, while rats in the MS group showed no significant difference (Figure 2B). Moreover, there was no significant difference between CUMS and MS+CUMS groups on PND60. 


\section{A}

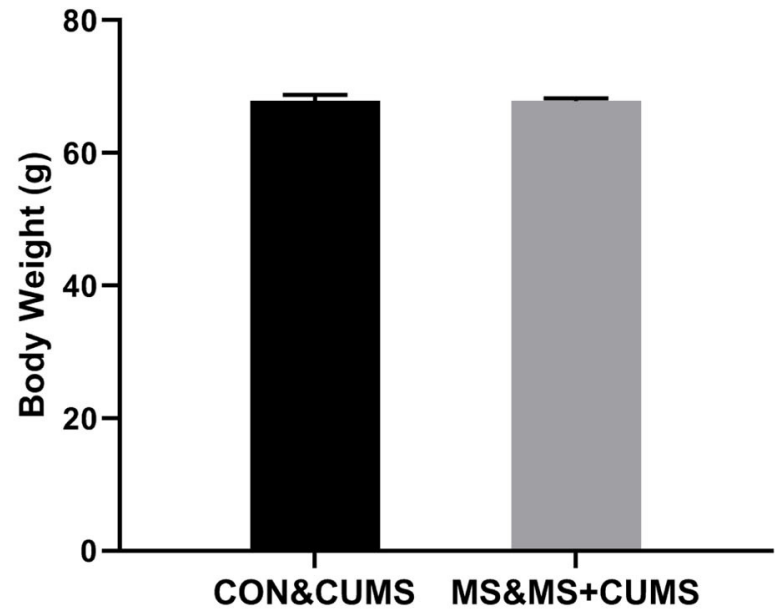

B

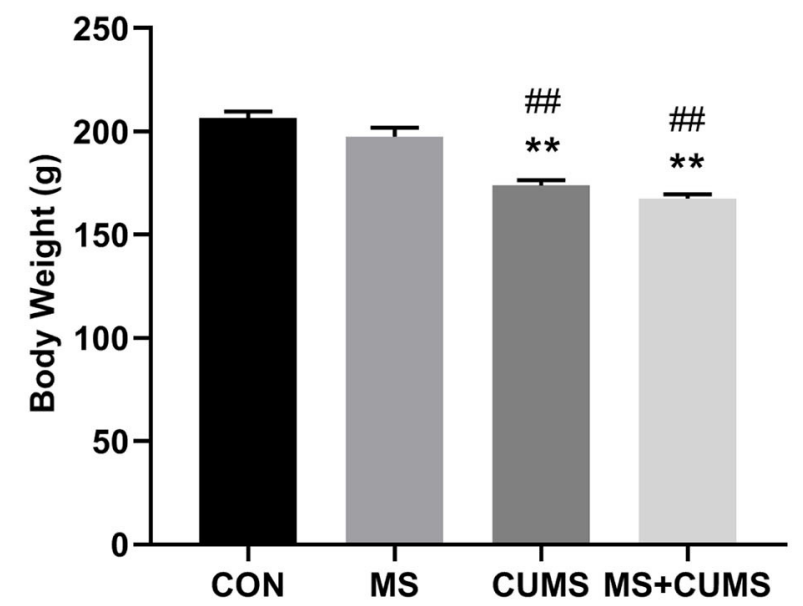

FIGURE 2 | Body weight record of rats on PND28 (A) $(n=16)$ and PND60 (B) $(n=8)$. The values represent the mean \pm SEM. ${ }^{* *} p<0.01$ vs. CON; \#\# $p<0.01$ vs. MS. CON, control; MS, maternal separation; CUMS, chronic unpredictable mild stress.

\section{The Effect of MS and/or CUMS on Behavioral Tests}

In SPT (Figure 3A), MS+CUMS group presented the lowest sucrose preference rate. MS showed a slight reduction in the sucrose preference rate, differing from the control group $\left[F_{(3,28)}\right.$ $=14.64, p<0.01]$. The CUMS group and MS+CUMS group presented a more noticeable decline in sucrose preference rate compare with the control group $\left[F_{(3,28)}=14.64, p<0.01\right.$ and $p<0.01)$. Importantly, the sucrose preference rate reduction was the most significant in rats that underwent MS as well as CUMS, surpassing the effect of MS only $\left[F_{(3,28)}=14.64, p<\right.$ 0.05]. By the way, there was no difference between CUMS group and MS+CUMS group.

In OFT (Figure 3B), although the MS group, CUMS group, and MS+CUMS group showed a noticeable reduction in central region time, the MS group showed no statistical difference compared to the control group, while the CUMS group $\left[F_{(3,28)}=\right.$ $6.51, p<0.05]$ and MS+CUMS group $\left[F_{(3,28)}=6.51, p<0.05\right]$ presented a significant reduction. Double stress of MS and CUMS led to the shortest total distance among four group (Figure 3C). The MS group showed no obvious decline in total distance, and the CUMS group presented a reduction with no statistical difference. However, the total distance revealed a significant difference in MS+CUMS group compared with the control group $\left[F_{(3,28)}=5.11, p<0.05\right]$ and also with the MS group $\left[F_{(3,28)}=\right.$ $5.11, p<0.05]$.

In ZMT, MS+CUMS group showed the least time spent in open areas (Figure 3D). The CUMS group $\left[F_{(3,28)}=13.18, p\right.$ $<0.01]$ as well as MS+CUMS group $\left[F_{(3,28)}=13.18, p<0.01\right]$ presented decreased time spent in open areas compared with the control group. Notably, double stress caused the most significant reduction in time spent in open areas, even less than MS only $\left[F_{(3,28)}=13.18, p<0.01\right]$ and CUMS only $\left[F_{(3,28)}=13.18, p<\right.$
0.05, not shown]. Incidentally, the CUMS group showed less time spent in open areas than the MS group $\left[F_{(3,28)}=13.18, p<0.05\right]$.

\section{The Effect of MS and/or CUMS on NissI Bodies}

According to Nissl staining, the neurons of the hippocampus in control group rats were in a large quantity and in a compact arrangement, while neurons in MS, CUMS, and MS+CUMS group rats were sparsely arranged and low in number, with neurons in the MS+CUMs group being the sparsest and the least in number (Figure 4C). Both in CA1 and DG of hippocampus, fewer Nissl positive cells were found in the MS group [CA1: $F_{(3,8)}$ $=21.50, p<0.01$; DG: $\left.F_{(3,8)}=21.80, p<0.01\right]$ and CUMS group [CA1: $F_{(3,8)}=21.50, p<0.01$; DG: $\left.F_{(3,8)}=21.80, p<0.01\right)$ compared to the control group (Figures 4A,B). Also, both in the CA1 and DG of the hippocampus, double stress of MS and CUMS exhibited fewer Nissl-positive cells than the control group [CA1: $F_{(3,8)}=21.50, p<0.01$; DG: $\left.F_{(3,8)}=21.80, p<0.01\right]$, which is even fewer than the MS only [CA1: $F_{(3,8)}=21.50, p<0.01$; DG: $\left.F_{(3,8)}=21.80, p<0.01\right]$ and CUMS only groups [CA1: $F_{(3,8)}=$ 21.50, $p<0.05$; DG: $F_{(3,8)}=21.80, p<0.01$, not shown].

\section{The Effect of MS and/or CUMS on Expressions of Synaptic Plasticity Proteins in Hippocampus}

Synaptic plasticity proteins were detected by western blot, Figure 5 shows that the expressions of PSD-95, GAP-43 and SYN in CUMS group [PSD-95: $F_{(3,8)}=5.54, p<0.05$; GAP-43: $F_{(3,8)}$ $=11.84, p<0.01$; SYN: $\left.F_{(3,8)}=8.60, p<0.01\right]$ and MS+CUMS group [PSD-95: $F_{(3,8)}=5.54, p<0.01$; GAP-43: $F_{(3,8)}=11.84$, $p<0.01$; SYN: $\left.F_{(3,8)}=8.60, p<0.01\right]$ were downregulated. The expressions of those proteins were significantly lower in MS+CUMS group than MS group [PSD-95: $F_{(3,8)}=5.54, p<$ 
A

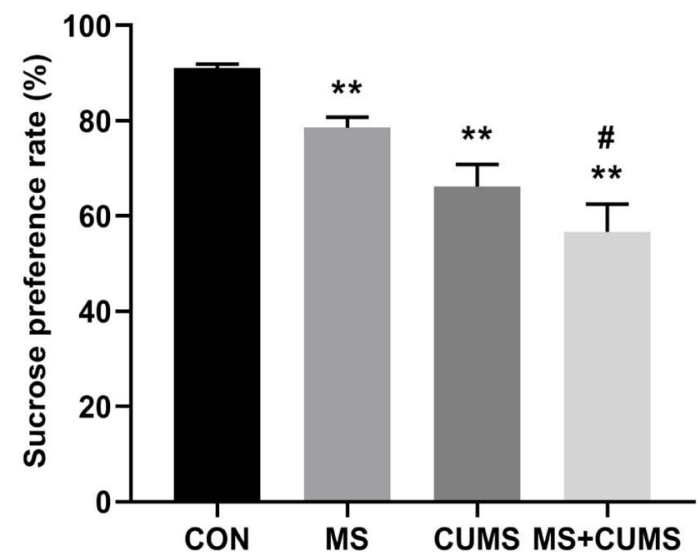

c

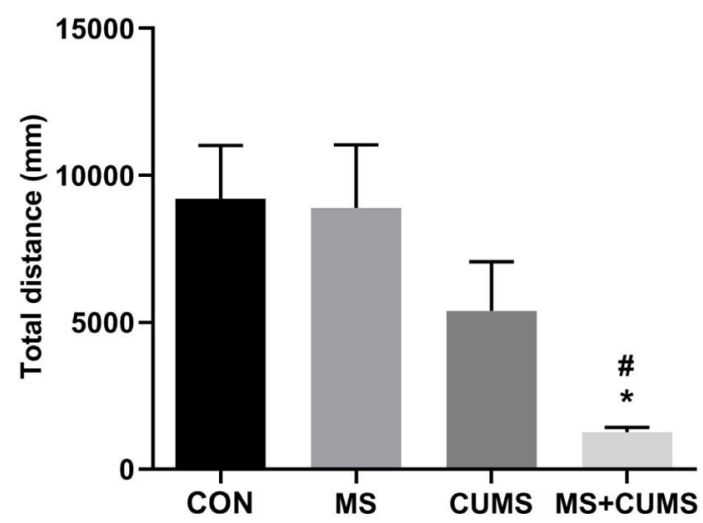

B

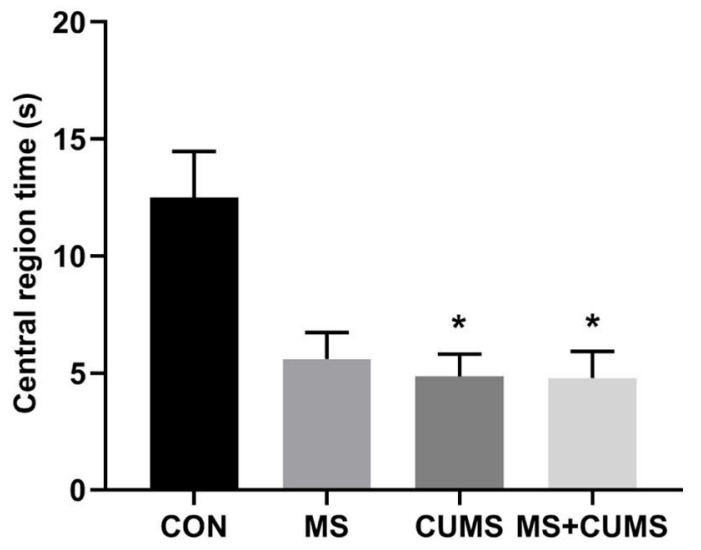

D

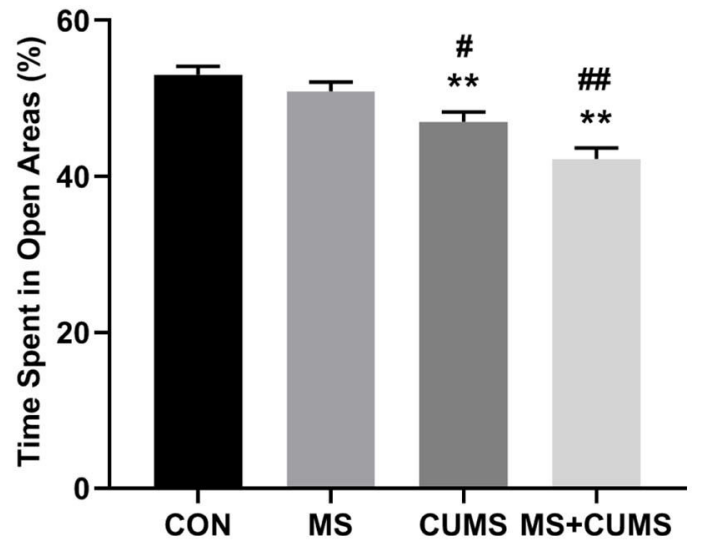

FIGURE 3 | The effects of MS and CUMS on behavior in female rats. After undergoing MS and/or CUMS, all groups of rats performed behavior tests. In the sucrose preference test, the sucrose preference rate was analyzed as factors (A); in the open field test, central region time (B) and total distance (C) were analyzed as factors; in the zero maze test, time spent in open areas was analyzed as factors (D). The values represent the mean $\pm \mathrm{SEM}, n=8 .{ }^{\star} p<0.05,{ }^{\star \star} p<0.01$ vs. CON, ${ }^{*} p<$ 0.05, ${ }^{\# \# ~} p<0.01$ vs. MS. CON, control; MS, maternal separation; CUMS, chronic unpredictable mild stress.

0.05; GAP-43: $F_{(3,8)}=11.84, p<0.01$; SYN $F_{(3,8)}=8.60, p<$ 0.05]. Here, the double stress of MS and CUMS led to the lowest expressions of those proteins among all groups (Figure 5A).

In order to further determined the expression of SYN, immunohistochemistry was detected (Figure 6). Both in CA1 (Figure 6A) and DG (Figure 6B) of hippocampus, SYN expression was radically diminished in MS group [CA1: $F_{(3,8)}$ $=45.45, p<0.05$; DG: $\left.F_{(3,8)}=14.89, p<0.01\right]$ and CUMS group [CA1: $F_{(3,8)}=45.45, p<0.01$; DG: $F_{(3,8)}=14.89, p$ $<0.01$ ] as well as MS+CUMS group [CA1: $F_{(3,8)}=45.45, p$ $<0.01$; DG: $\left.F_{(3,8)}=14.89, p<0.01\right]$ compared with control group. MS+CUMS group presented the least percentage of the SYN-positive area both in CA1 and DG (Figure 6C). Both in CA1 and DG of the hippocampus, double stress of MS and CUMS led to lower SYN expression than the MS group [CA1: $F_{(3,8)}=45.45, p<0.05$; DG: $\left.F_{(3,8)}=14.89, p<0.01\right]$.

\section{DISCUSSION}

In the present study, we examined the effects of MS, CUMS, and MS combined with CUMS on body weight, depression-like and anxiety-like behaviors, and expression of synapse-associated proteins. We found that with the experience of MS at an early age and CUMS in adolescence, adult female rats showed very intense depression-like and anxiety-like behaviors, which were more severe than those who underwent only MS or only CUMS. Moreover, depression-like and anxiety-like behaviors were related to the abnormal expressions of synapse-associated proteins, including PSD-95, GAP-43, and SYN. This study demonstrated for the first time that MS combined with CUMS induced more critical depression-like and anxiety-like behaviors than MS only or CUMS only in female rats, and the 
A

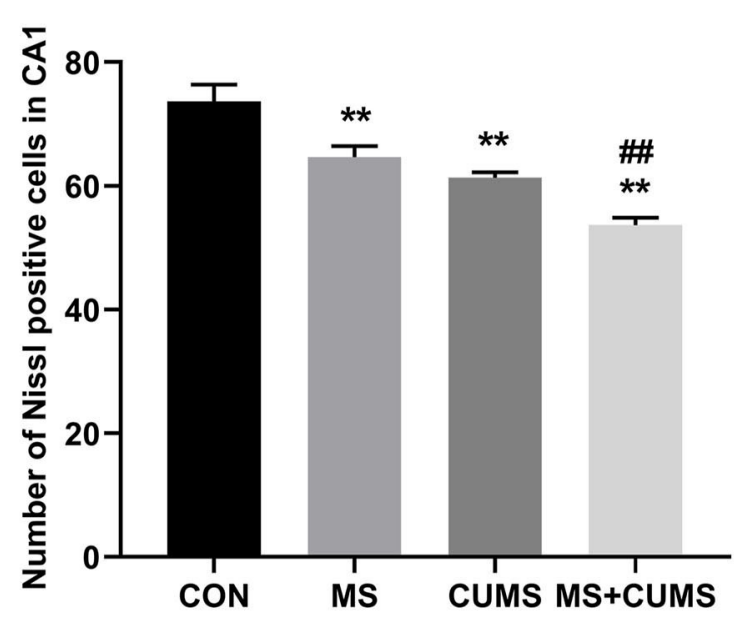

B

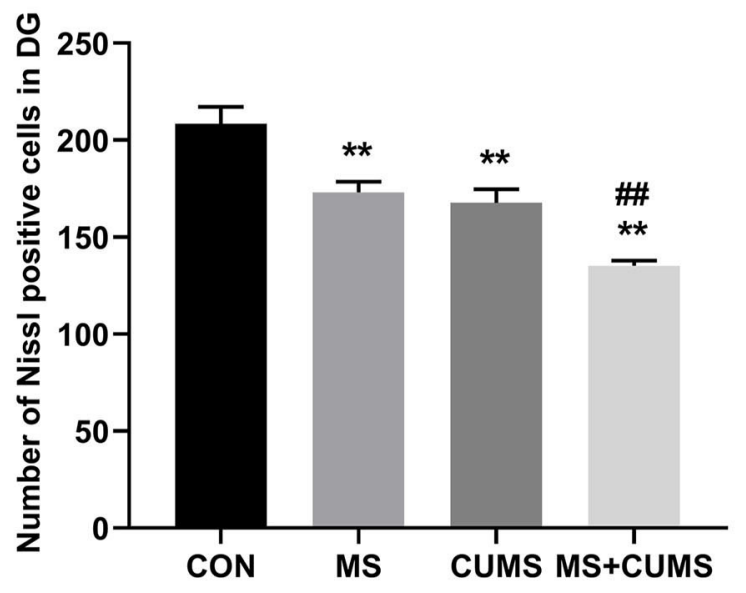

C

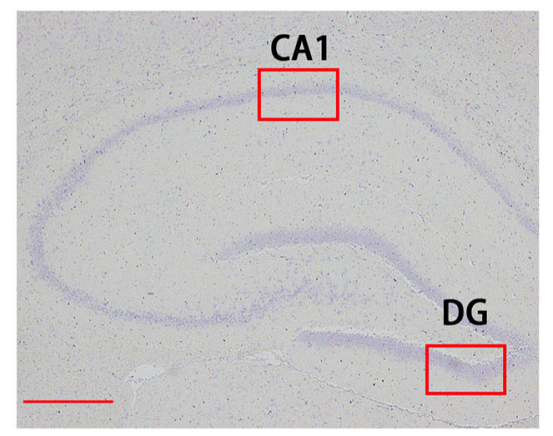

\section{CA1}
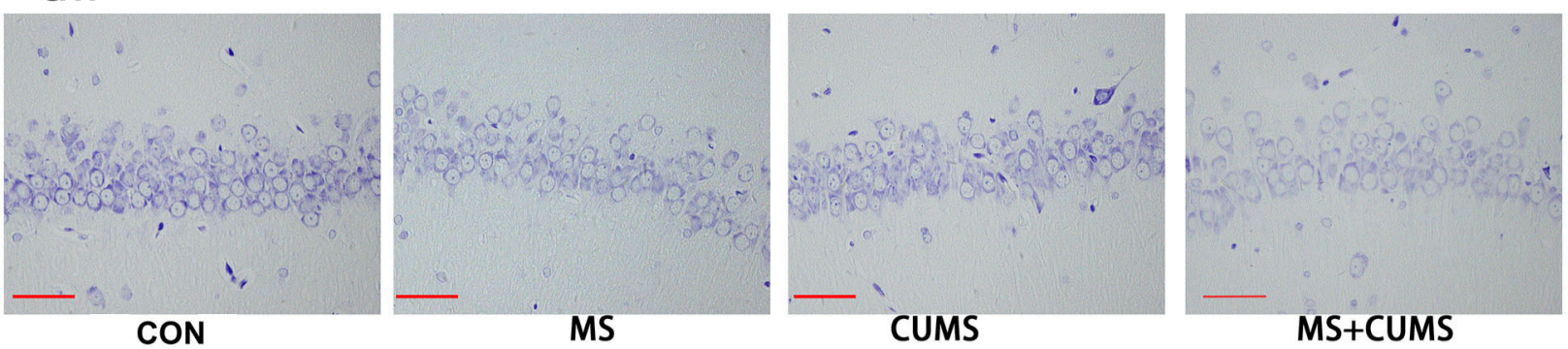

DG
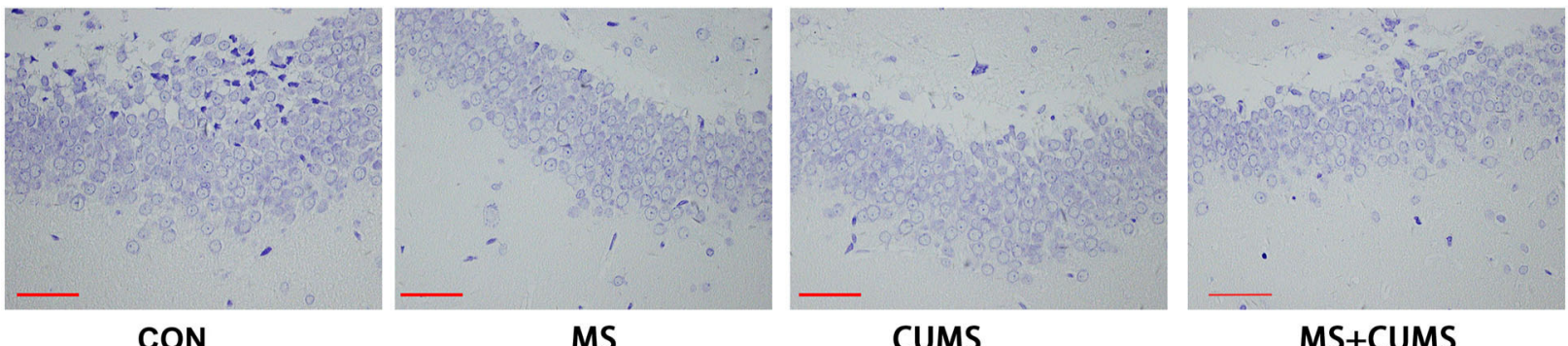

FIGURE 4 | Nissl staining in the hippocampus. (A) Number of Nissl positive cells in CA1; (B) Number of Nissl positive cells in DG; (C) Representative

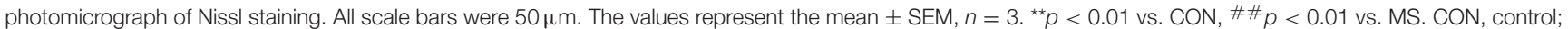
MS, maternal separation; CUMS, chronic unpredictable mild stress; CA1, cornu ammonis 1; DG, dentate gyrus. 
A CON MS CUMS MS+CUMS

PSD-95

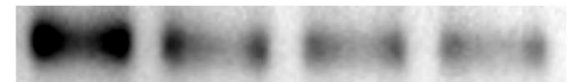

GAP-43

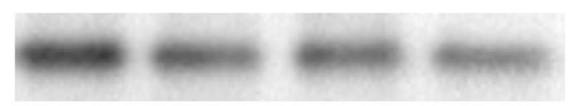

SYN

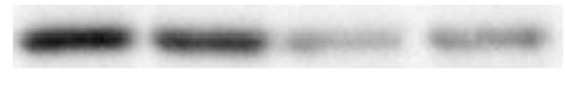

Tubulin

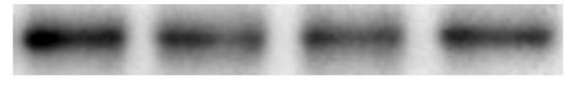

$55 \mathrm{kD}$

$38 \mathrm{kD}$

C

\section{5kD}

$43 k D$

D
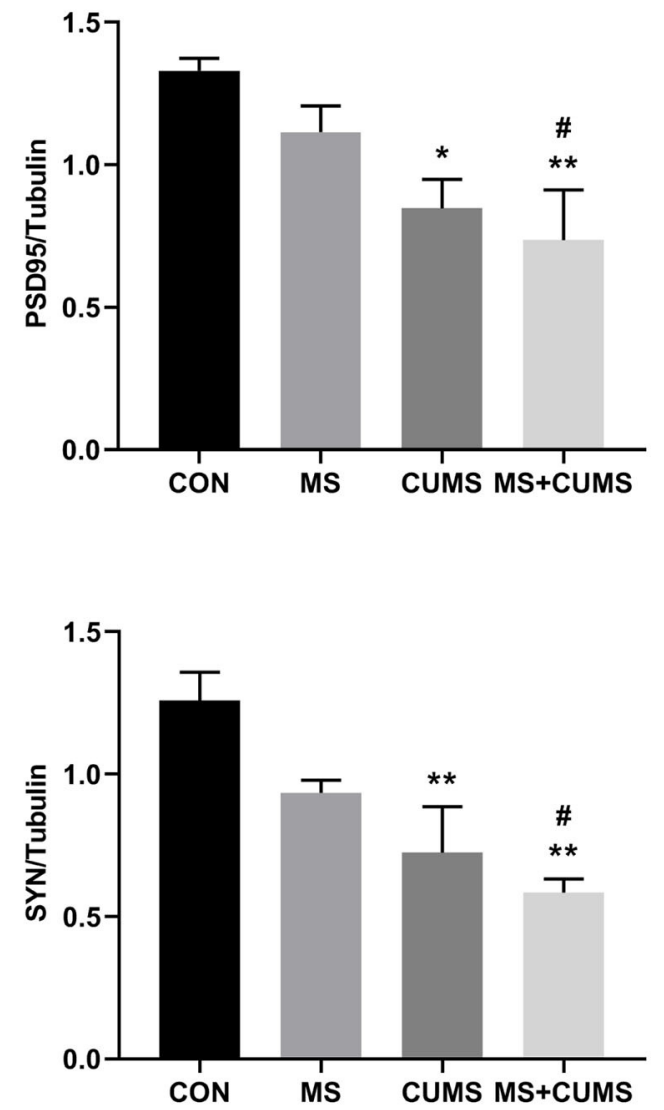

FIGURE 5 | Western blot analysis. The expressions of synaptic plasticity proteins were determined by western blot (A), including PSD-95 (B), GAP-43 (C), and SYN (D). The values represent the mean \pm SEM, $n=5$. ${ }^{*} p<0.05,{ }^{* *} p<0.01$ vs. CON, $\# p<0.05$, $\# \# p<0.01$ vs. MS. CON, control; MS, maternal separation; CUMS, chronic unpredictable mild stress.

impairment of hippocampal synaptic plasticity may be the potential mechanism.

In this study, we observed the effects of MS, CUMS, and MS combined CUMS on body weight and behaviors of rats. Knowing that losing weight could be one of the symptoms of depression (27), the treatment of CUMS and MS+CUMS led to an obvious loss in weight in our study, which indicated that animals were in a progression of depression-like behavior, and this was consistent with previous studies $(28,29)$. Gracia-Rubio et al. reported that maternal separation caused emotional alterations but had no impact on body weight (30). Consisted with this report, our study showed that MS had no effect on body weight, while CUMS had.

Here, we revealed that rats that underwent MS showed significantly increased depression-like and anxiety-like behaviors after exposure to a CUMS environment. These findings are in agreement with previous studies showing that stress at an early stage is an important factor in the development of depression-like behaviors when exposed to adult stress, and this resulted in neurodevelopmental abnormalities through stress
(31). This is consistent with studies showing that early life experience is associated with susceptibility to depression (3235). In our study, only MS treatment of rats did not lead to anxiety-like behavior. Similarly, Chen's study showed anxietylike behavior did not occur in MS rats (36). However, on the contrary, MS could present anxiety-like behavior in some studies $(37,38)$, we cannot definitely explain the different behavioral performances owing to different MS protocol and animals. So far, the opinion that a longer period of MS could induce anxiety-like behavior was confirmed in Arias' study (39). Interestingly, from the result of Susana Roque, only rats in $\mathrm{MS}_{2-15}$ group (MS from PND2-15) displayed depressive and anxiety-like behaviors, while rats in $\mathrm{MS}_{7-20}$ group (MS from PND7-20) did not display these (40). These questions need more experiment to verify.

Nissl staining is often used to detect the activity of neurons and impairment of neurons would lead to the reduction of Nissl bodies $(41,42)$. A small amount of Nissl positive cells was found in the hippocampus of rats underwent MS as well as CUMS. In 
A

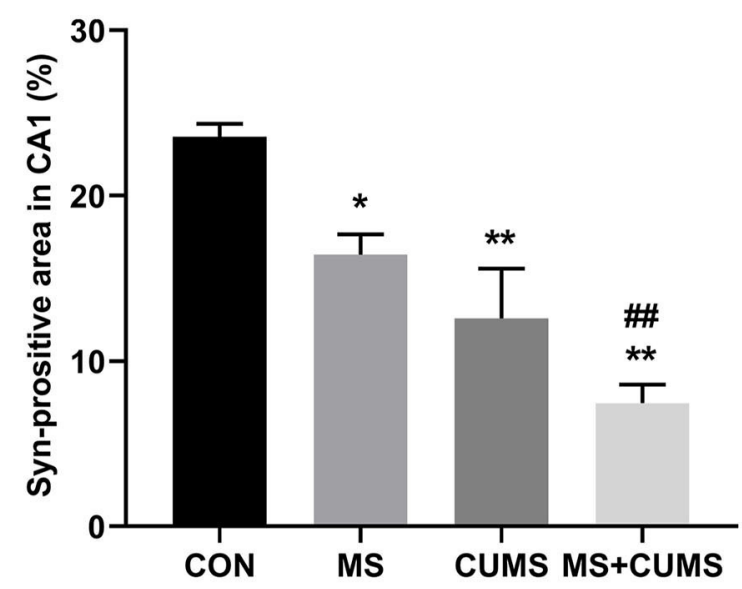

B

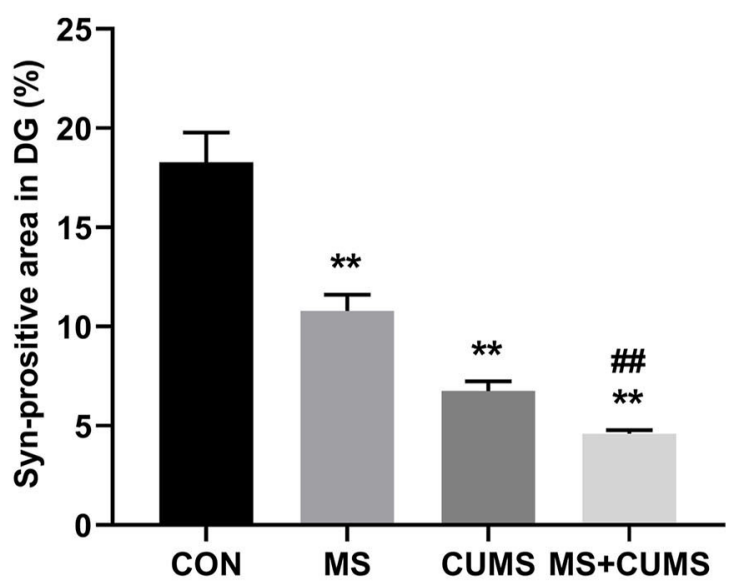

c

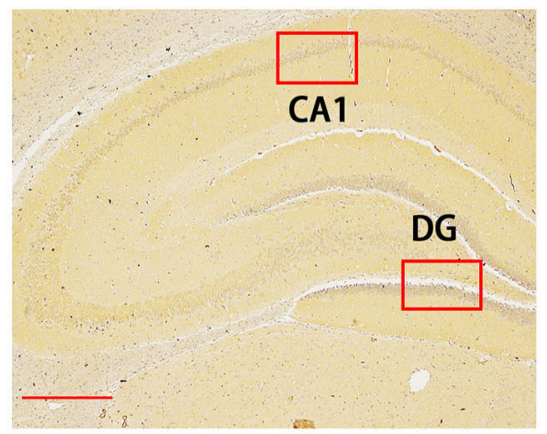

\section{CA1}

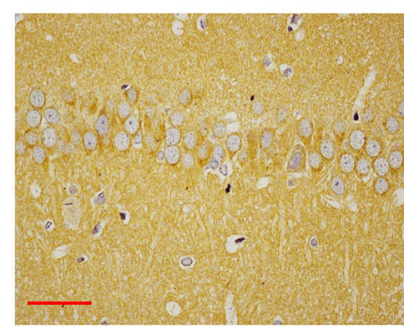

CON

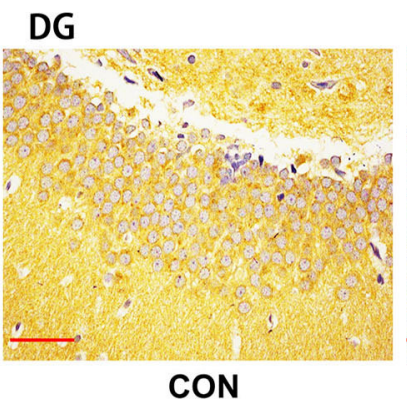

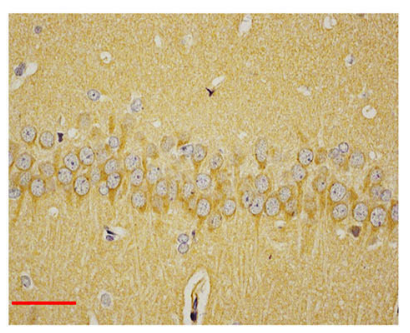

MS

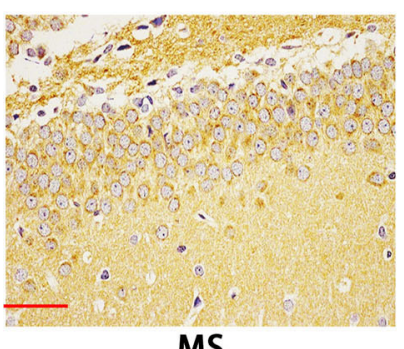

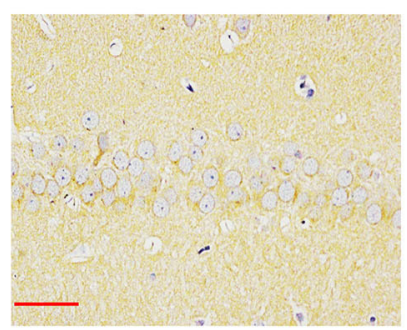

CUMS

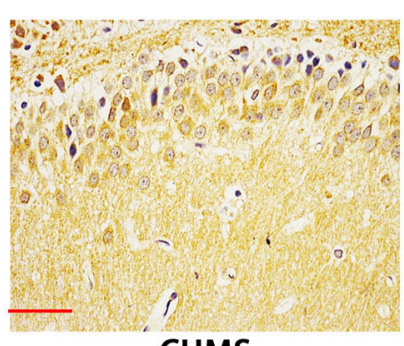

CUMS

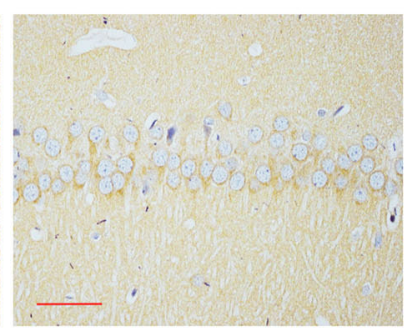

MS+CUMS

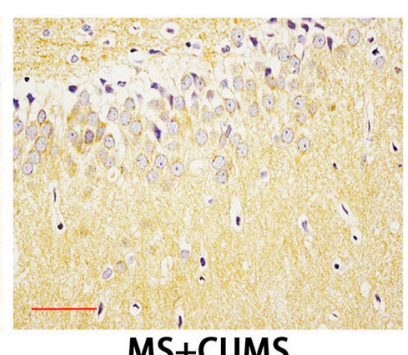

FIGURE 6 | Anti-SYN immunohistochemistry analysis in the hippocampus. (A) Syn-positive area in CA1; (B) Syn-positive area in DG; (C) representative photomicrograph of immunohistochemistry. All scale bars were $50 \mu \mathrm{m}$. The values represent the mean $\pm \mathrm{SEM}, n=3$. ${ }^{*} p<0.05,{ }^{* *} p<0.01$ vs. CON, ${ }^{\# \#} p<0.01$ vs. MS. CON, control; MS, maternal separation; CUMS, chronic unpredictable mild stress, CA1, cornu ammonis 1; DG, dentate gyrus. 
other words, those stress may affect the activity of neurons. This conjecture is further confirmed by the following experiments.

Clinical studies have found the reduction of synaptic signaling proteins expressed in the brain regions (such as the hippocampus) with major depressive disorder patients $(43,44)$. SYN, PSD-95, and GAP-43 are considered to be key proteins affecting synaptic plasticity $(45,46)$ as well as normal signaling between neurons in the central nervous system (47). SYN is a vesicle protein of presynaptic membrane, which is closely linked to the regulation of synaptic structure and functional plasticity, which widely exists in the synaptic membrane of neurons (48) as well as rapidly recruits to the presynaptic end in response to presynaptic neuronal activity (49). In our study, low expression of SYN was detected in the hippocampus of rats that underwent MS and CUMS. Studies have shown that abnormal SYN expression is associated with depression (50). The atrophy of dendrites in the vertebral hippocampal neurons and the downregulation of SYN expression existed in chronic stress and depression (51).

PSD-95 is one of the postsynaptic densities (PSDs), which plays a key role in synaptic plasticity signal transduction $(52,53)$. Studies have found that most neurological diseases, such as depression, show abnormal expression of PSD-95 (54, 55). GAP-43 is located on the growth cone of the axon and participates in the synaptic plasticity of the nervous system $(56,57)$. In addition, GAP-43 is essential for synaptic plasticity, axon elongation, and nerve germination during the development and maturation of neurons in adult rats (58, 59). Our results are similar to $\mathrm{Li}$ and Yang et al. in that the expression of GAP43 in rats with depression-like and anxiety-like behaviors is significantly downregulated (60). The results showed that the expression of PSD-95 had the same trend as GAP-43. This evidence further suggested that MS combined CUMS had an effect on the impairment of synaptic plasticity. Moreover, when fewer synaptic plasticity proteins were expressed, the rats displayed more serious depression-like and anxiety-like behavior.

As the model of early MS combined with adolescence, CUMS produced critical depression-like and anxiety-like behavior as well as synaptic plasticity impairment, our finding may help to provide a novel convincing depression model. However, what is the specific mechanism? Further study would verify whether

\section{REFERENCES}

1. Mathers CD, Loncar D. Projections of global mortality and burden of disease from 2002 to 2030. PLoS Med. (2006) 3:e442. doi: 10.1371/journal.pmed.0030442

2. Caspi A. Influence of life stress on depression: moderation by a polymorphism in the 5-HTT gene. Science. (2003) 301:386-9. doi: 10.1126/science.1083968

3. Berton O, Nestler EJ. New approaches to antidepressant drug discovery: beyond monoamines. Nat Rev Neurosci. (2006) 7:137-51. doi: 10.1038/nrn1846

4. Felitti VJ, Anda RF, Nordenberg D, Williamson DF, Spitz AM, Edwards V, et al. Relationship of childhood abuse and household dysfunction to many of the leading causes of death in adults: the adverse childhood experiences (ACE) study. Am J Prev Med. (2019) 56:774-86. doi: 10.1016/j.amepre.2019.04.001 synaptic plasticity proteins be the important targets for antidepressant interventions.

\section{CONCLUSION}

In conclusion, we demonstrated that early MS combined with adolescent CUMS in female rats could induce more critical depression-like and anxiety-like behaviors. The expressions of synaptic-related proteins and the impairment of synaptic plasticity may be the potential mechanisms.

\section{DATA AVAILABILITY STATEMENT}

The datasets generated for this study are available on request to the corresponding author.

\section{ETHICS STATEMENT}

The animal study was reviewed and approved by Animal Experimentation Committee at Guangzhou University of Chinese Medicine.

\section{AUTHOR CONTRIBUTIONS}

$\mathrm{JH}$ wrote the manuscript and conducted animal experiment. CS and RY conducted animal and analysis experiment. YS analyzed data and corrected the manuscript. WL controlled all work and revised manuscript. All authors contributed to the article and approved the submitted version.

\section{FUNDING}

This work was supported by the National Natural Science Foundation of China (No. 81873271, 81573912, 81573638, 82074505), Guangdong Natural Science Foundation of China (No. 2017A030313666), Guangzhou Science Technology and Innovation Commission Technology Research Projects (No. 201805010005), Key laboratory project of colleges and universities in Guangdong province (No. 2019KSYS005), and Guangdong province science and technology plan international cooperation project (No. 2020A0505100052).
5. Spritzer MD, Fox EC, Larsen GD, Batson CG, Wagner BA, Maher J, et al. Testosterone influences spatial strategy preferences among adult male rats. Horm Behav. (2013) 63:800-12. doi: 10.1016/j.yhbeh.2013.03.018

6. Smarr BL, Grant AD, Zucker I, Prendergast BJ, Kriegsfeld LJ. Sex differences in variability across timescales in BALB/c mice. Biol Sex Differ. (2017) 8:7. doi: 10.1186/s13293-016-0125-3

7. Maghami S, Zardooz H, Khodagholi F, Binayi F, Ranjbar SR, Hedayati M, et al. Correction: maternal separation blunted spatial memory formation independent of peripheral and hippocampal insulin content in young adult male rats. PLoS ONE. (2019) 14:e210893. doi: 10.1371/journal.pone. 0210893

8. Kuehner C. Why is depression more common among women than among men? Lancet Psychiatry. (2017) 4:14658. doi: 10.1016/S2215-0366(16)30263-2 
9. Li SH, Graham BM. Why are women so vulnerable to anxiety, traumarelated and stress-related disorders? Lancet Psychiatry. (2017) 4:7382. doi: 10.1016/S2215-0366(16)30358-3

10. Qiao H, Li MX, Xu C, Chen HB, An SC, Ma XM, et al. Dendritic spines in depression: what we learned from animal models. Neural Plast. (2016) 2016:8056370. doi: 10.1155/2016/8056370

11. Garber J, Brunwasser SM, Zerr AA, Schwartz KT, Sova K, Weersing VR, et al. Treatment and prevention of depression and anxiety in youth: test of cross-over effects. Depress Anxiety. (2016) 33:939-59. doi: 10.1002/da.22519

12. Price JL, Drevets WC. Neurocircuitry of mood disorders. Neuropsychopharmacology. (2010) 35:192-216. doi: 10.1038/npp.2009.104

13. MacQueen G, Frodl T. The hippocampus in major depression: evidence for the convergence of the bench and bedside in psychiatric research? $\mathrm{Mol}$ Psychiatry. (2011) 16:252-64. doi: 10.1038/mp.2010.80

14. Duman RS, Aghajanian GK. Synaptic dysfunction in depression: potential therapeutic targets. Science. (2012) 338:68-72. doi: 10.1126/science.1222939

15. Shen J, Li Y, Qu C, Xu L, Sun H, Zhang J, et al. The enriched environment ameliorates chronic unpredictable mild stress-induced depressive-like behaviors and cognitive impairment by activating the SIRT1/miR-134 signaling pathway in hippocampus. J Affect Disord. (2019) 248:81-90. doi: 10.1016/j.jad.2019.01.031

16. Petrik D, Lagace DC, Eisch AJ. The neurogenesis hypothesis of affective and anxiety disorders: are we mistaking the scaffolding for the building? Neuropharmacology. (2012) 62:21-34. doi: 10.1016/j.neuropharm.2011.09.003

17. Zahorodna A, Tokarski K, Bijak M. Electrophysiologic tests for testing the effects of antidepressant drugs and corticosterone on reactivity of serotonin receptors in the hippocampus. Postepy Hig Med Dosw. (2000) 54:391-401.

18. Kim JJ, Lee HJ, Welday AC, Song E, Cho J, Sharp PE, et al. Stress-induced alterations in hippocampal plasticity, place cells, and spatial memory. Proc Natl Acad Sci USA. (2007) 104:18297-302. doi: 10.1073/pnas.0708644104

19. Zhao L, Ren H, Gu S, Li X, Jiang C, Li J, et al. rTMS ameliorated depressivelike behaviors by restoring HPA axis balance and prohibiting hippocampal neuron apoptosis in a rat model of depression. Psychiatry Res. (2018) 269:12633. doi: 10.1016/j.psychres.2018.08.017

20. Pittenger C, Duman RS. Stress, depression, and neuroplasticity: a convergence of mechanisms. Neuropsychopharmacology. (2008) 33:88-109. doi: 10.1038/sj.npp.1301574

21. Duman RS, Aghajanian GK, Sanacora G, Krystal JH. Synaptic plasticity and depression: new insights from stress and rapid-acting antidepressants. Nat Med. (2016) 22:238-49. doi: 10.1038/nm.4050

22. Menard C, Hodes GE, Russo SJ. Pathogenesis of depression: insights from human and rodent studies. Neuroscience. (2016) 321:138-62. doi: 10.1016/j.neuroscience.2015.05.053

23. Liu W, Xue X, Xia J, Liu J, Qi Z. Swimming exercise reverses CUMS-induced changes in depression-like behaviors and hippocampal plasticity-related proteins. J Affect Disord. (2018) 227:126-35. doi: 10.1016/j.jad.2017.10.019

24. Shen C, Cao K, Cui S, Cui Y, Mo H, Wen W, et al. SiNiSan ameliorates depression-like behavior in rats by enhancing synaptic plasticity via the CaSR-PKC-ERK signaling pathway. Biomed Pharmacother. (2020) 124:109787. doi: 10.1016/j.biopha.2019.109787

25. Willner P, Towell A, Sampson D, Sophokleous S, Muscat R. Reduction of sucrose preference by chronic unpredictable mild stress, and its restoration by a tricyclic antidepressant. Psychopharmacology. (1987) 93:35864. doi: 10.1007/BF00187257

26. Zhu H, Tao Y, Wang T, Zhou J, Yang Y, Cheng L, et al. Long-term stability and characteristics of behavioral, biochemical, and molecular markers of three different rodent models for depression. Brain Behav. (2020) 10:e1508. doi: 10.1002/brb3.1508

27. Sawamura J, Morishita S, Ishigooka J. Symmetrical treatment of "Diagnostic and Statistical Manual of Mental Disorders, Fifth Edition," for major depressive disorders. Source Code Biol Med. (2016) 11:1. doi: 10.1186/s13029-015-0041-7

28. Jiang Y, Wang Y, Sun X, Lian B, Sun H, Wang G, et al. Short- and long-term antidepressant effects of ketamine in a rat chronic unpredictable stress model. Brain Behav. (2017) 7:e749. doi: 10.1002/brb3.749

29. Dong H, Cong W, Guo X, Wang Y, Tong S, Li Q, et al. beta-asarone relieves chronic unpredictable mild stress induced depression by regulating the extracellular signal-regulated kinase signaling pathway. Exp Ther Med. (2019) 18:3767-74. doi: 10.3892/etm.2019.8018

30. Gracia-Rubio I, Moscoso-Castro M, Pozo OJ, Marcos J, Nadal R, Valverde $\mathrm{O}$, et al. Maternal separation induces neuroinflammation and long-lasting emotional alterations in mice. Prog Neuropsychopharmacol Biol Psychiatry. (2016) 65:104-17. doi: 10.1016/j.pnpbp.2015.09.003

31. Pena CJ, Smith M, Ramakrishnan A, Cates HM, Bagot RC, Kronman HG, et al. Early life stress alters transcriptomic patterning across reward circuitry in male and female mice. Nat Commun. (2019) 10:5098. doi: 10.1038/s41467-019-13085-6

32. Marais L, van Rensburg SJ, van Zyl JM, Stein DJ, Daniels WM. Maternal separation of rat pups increases the risk of developing depressive-like behavior after subsequent chronic stress by altering corticosterone and neurotrophin levels in the hippocampus. Neurosci Res. (2008) 61:10612. doi: 10.1016/j.neures.2008.01.011

33. Jia R, Tai F, An S, Zhang X, Broders H. Effects of neonatal paternal deprivation or early deprivation on anxiety and social behaviors of the adults in mandarin voles. Behav Processes. (2009) 82:271-8. doi: 10.1016/j.beproc.2009.07.006

34. Rentesi G, Antoniou K, Marselos M, Fotopoulos A, Alboycharali J, Konstandi $\mathrm{M}$, et al. (2010). Long-term consequences of early maternal deprivation in serotonergic activity and HPA function in adult rat. Neurosci Lett. 480:711. doi: 10.1016/j.neulet.2010.04.054

35. Jaric I, Rocks D, Cham H, Herchek A, Kundakovic M. Sex and estrous cycle effects on anxiety- and depression-related phenotypes in a two-hit developmental stress model. Front Mol Neurosci. (2019) 12:74. doi: 10.3389/fnmol.2019.00074

36. Zheng $\mathrm{Y}$, He J, Guo L, Yao L, Zheng X, Yang Z, et al. Transcriptome analysis on maternal separation rats with depression-related manifestations ameliorated by electroacupuncture. Front Neurosci. (2019) 13:314. doi: 10.3389/fnins.2019.00314

37. Park SS, Park HS, Kim CJ, Baek SS, Kim TW. Exercise attenuates maternal separation-induced mood disorder-like behaviors by enhancing mitochondrial functions and neuroplasticity in the dorsal raphe. Behav Brain Res. (2019) 372:112049. doi: 10.1016/j.bbr.2019.112049

38. Liu C, Hao S, Zhu M, Wang Y, Zhang T, Yang Z, et al. (2018). Maternal separation induces different autophagic responses in the hippocampus and prefrontal cortex of adult rats. Neuroscience. 374:287-94. doi: 10.1016/j.neuroscience.2018.01.043

39. Banqueri M, Mendez M, Arias JL. Behavioral effects in adolescence and early adulthood in two length models of maternal separation in male rats. Behav Brain Res. (2017) 324:77-86. doi: 10.1016/j.bbr.2017.02.006

40. Roque S, Mesquita AR, Palha JA, Sousa N, Correia-Neves M. The behavioral and immunological impact of maternal separation: a matter of timing. Front Behav Neurosci. (2014) 8:192. doi: 10.3389/fnbeh.2014.00192

41. Su YT, Guo YB, Cheng YP, Zhang X, Xie XP, Chang YM, et al. Hyperbaric oxygen treatment ameliorates hearing loss and auditory cortex injury in noise exposed mice by repressing local ceramide accumulation. Int J Mol Sci. (2019) 20:1675. doi: 10.3390/ijms20194675

42. Yamauchi A, Tone T, Sugimoto K, Seok LH, Kaku T, Tohda C, et al. Porcine placental extract facilitates memory and learning in aged mice. Food Sci Nutr. (2019) 7:2995-3005. doi: 10.1002/fsn3.1156

43. Kang HJ, Voleti B, Hajszan T, Rajkowska G, Stockmeier CA, Licznerski P, et al. Decreased expression of synapse-related genes and loss of synapses in major depressive disorder. Nat Med. (2012) 18:1413-7. doi: 10.1038/nm.2886

44. Zhao J, Bao AM, Qi XR, Kamphuis W, Luchetti S, Lou JS, et al. Gene expression of GABA and glutamate pathway markers in the prefrontal cortex of non-suicidal elderly depressed patients. J Affect Disord. (2012) 138:494502. doi: 10.1016/j.jad.2012.01.013

45. Sifonios L, Trinchero M, Cereseto M, Ferrero A, Cladouchos ML, Macedo GF, et al. An enriched environment restores normal behavior while providing cytoskeletal restoration and synaptic changes in the hippocampus of rats exposed to an experimental model of depression. Neuroscience. (2009) 164:929-40. doi: 10.1016/j.neuroscience.2009.08.059

46. Li H, Linjuan-Li, Wang Y. G-CSF improves CUMS-induced depressive behaviors through downregulating Ras/ERK/MAPK signaling pathway. Biochem Biophys Res Commun. (2016) 479:827-32. doi: 10.1016/j.bbrc.2016.09.123 
47. Marco EM, Ballesta JA, Irala C, Hernandez MD, Serrano ME, Mela V, et al. Sex-dependent influence of chronic mild stress (CMS) on voluntary alcohol consumption; study of neurobiological consequences. Pharmacol Biochem Behav. (2017) 152:68-80. doi: 10.1016/j.pbb.2016.11.005

48. Kwon SE, Chapman ER. Synaptophysin regulates the kinetics of synaptic vesicle endocytosis in central neurons. Neuron. (2011) 70:847-54. doi: 10.1016/j.neuron,.2011.04.001

49. Basu J, Siegelbaum SA. The corticohippocampal circuit, synaptic plasticity, and memory. Cold Spring Harbor Perspectiv Biol. (2015) 7:a21733. doi: 10.1101/cshperspect.a021733

50. Christoffel DJ, Golden SA, Russo SJ. Structural and synaptic plasticity in stress-related disorders. Rev Neurosci. (2011) 22:535-49. doi: 10.1515/RNS.2011.044

51. Kim JJ, Diamond DM. The stressed hippocampus, synaptic plasticity and lost memories. Rev Neurosci. (2002) 3:453-62. doi: 10.1038/nrn849

52. Scannevin RH, Huganir RL. Postsynaptic organization and regulation of excitatory synapses. Nat Rev Neurosci. (2000) 1:133-41. doi: 10.1038/35039075

53. Rapp S, Baader M, Hu M, Jennen-Steinmetz C, Henn FA, Thome J, et al. Differential regulation of synaptic vesicle proteins by antidepressant drugs. Pharmacogenomics J. (2004) 4:110-3. doi: 10.1038/sj.tpj.6500229

54. Wegener G, Harvey BH, Bonefeld B, Muller HK, Volke V, Overstreet DH, et al. Increased stress-evoked nitric oxide signalling in the Flinders sensitive line (FSL) rat: a genetic animal model of depression. Int J Neuropsychopharmacol. (2010) 13:461-73. doi: 10.1017/S1461145709990241

55. Ting JT, Peça J, Feng G. Functional consequences of mutations in postsynaptic scaffolding proteins and relevance to psychiatric disorders. Ann Rev Neurosci. (2012) 35:49-71. doi: 10.1146/annurev-neuro-062111150442
56. Grasselli G, Strata P. Structural plasticity of climbing fibers and the growth-associated protein GAP-43. Front Neural Circuits. (2013) 7:25. doi: $10.3389 /$ fncir.2013.00025

57. Morita S, Miyata S. Synaptic localization of growth-associated protein 43 in cultured hippocampal neurons during synaptogenesis. Cell Biochem Funct. (2013) 31:400-11. doi: 10.1002/cbf.2914

58. Almawi AW, Matthews LA, Larasati Myrox P, Boulton S, Lai C, Guarne A, et al. "AND" logic gates at work: crystal structure of Rad53 bound to Dbf4 and Cdc7. Sci Rep. (2016) 6:34237. doi: 10.1038/srep34237

59. Hung CC, Lin CH, Chang H, Wang CY, Lin SH, Hsu PC, et al. Astrocytic GAP43 induced by the TLR4/NF-kappaB/STAT3 axis attenuates astrogliosismediated microglial activation and neurotoxicity. J Neurosci. (2016) 36:202743. doi: 10.1523/JNEUROSCI.3457-15.2016

60. Li Q, Yang D, Wang J, Liu L, Feng G, Li J, et al. Reduced amount of olfactory receptor neurons in the rat model of depression. Neurosci Lett. (2015) 603:48-54. doi: 10.1016/j.neulet.2015.07.007

Conflict of Interest: The authors declare that the research was conducted in the absence of any commercial or financial relationships that could be construed as a potential conflict of interest.

Copyright (c) 2021 Huang, Shen, Ye, Shi and Li. This is an open-access article distributed under the terms of the Creative Commons Attribution License (CC BY). The use, distribution or reproduction in other forums is permitted, provided the original author(s) and the copyright owner(s) are credited and that the original publication in this journal is cited, in accordance with accepted academic practice. No use, distribution or reproduction is permitted which does not comply with these terms. 